\title{
Karaji's L-Summing Method and the Generalized Ibn al-Haytham Recursive Formula
}

\author{
Hossein TeImoori FAAL (D) \\ Department of Mathematics and Computer Science, Allameh Tabataba'i University, Tehran, Iran.
}

Received: 09-04-2020 • Accepted: 22-04-2021

\begin{abstract}
Aвstract. In this paper, we first review two folkloric summation techniques due to two Islamic medieval scholars Karaji and Ibn al-Haytham by emphasizing on their higher-dimensional extensions. We also include several interesting examples of algebraic identities obtained form the generalized Ibn al-Haytham formulas and Karaji's L-summing method. Finally, we will also show the interplay between these two different methods of summation.
\end{abstract}

2010 AMS Classification: 35C20, 35D10.

Keywords: Karaji's $L$-summing method, the generalized Ibn al-Haytham formula, summation techniques, sums of powers, harmonic numbers.

\section{INTRODUCTION}

There is no doubt that recursive formulas play an essential role in computational mathematics and computer science. Those recursive formulations can be used to obtain many interesting combinatorial summations and identities in a systematic way. Considering the fact that the mathematical theory of the analysis of algorithms covers mainly binomial identities, recurrence relations, operator methods and asymptotic analysis [1], it is clear that how important are learning summation techniques for analyzing the worst-case running time of many combinatorial algorithms in computer science descipline.

In this paper while reviewing two seemingly different summation techniques originally developed by two Islamic medieval scholars and their generalizations, we also explain the interconnection between them. The first formulation is due to Karaji [6] which is a method of summation based on the geometric idea and the second one is due to Ibn al-Haytham [3] which has a recursive nature and originally developed for computing sums of powers of integers. It is important to note that because of the role of developing effective ways of teaching powerful ideas in the design and analysis of algorithms, visual proof techniques like Karaji's L-summing method may have a great impact in computer science education specially in undergraduate level. Thus, visualizing the combinatorial summations proofs can be considered as another goal of this paper.

The paper is organized, as follows. The next section provides a quick review of Karaji's L-summing method for summations mainly based on [2]. In particular, we present a three-dimensional generalization of the so called $L$ summing equation (see Section 2) which can be generalized for any higher dimension based on the idea of the inclusionexclusion formula. We also provide lower-dimensional specializations of all those higher-dimensional formulas besides mentioning some sample examples of their applications. Then in Section 3, we also quickly review the generalized Ibn al-Haytham summation formula mainly based on the reference [4]. We also obtain several interesting variants of this

Email address: hossein.teimoori@atu.ac.ir (H.T. Faal) 
summation formula for higher-dimensional cases. Many examples are also provide for those summations. Finally, in Section 4, we investigate the interplay between these two seemingly diferent summation approaches. Indeed, we show that by appropriate choices of changing variables one can transform the generalized Ibn al-Haytham formula to the generalized $L$-summing equation and vice versa.

\section{KaraJI's L-Summing Method}

About 1000 years ago, an interesting geometric-based method was used by M. Karaji [6] an Iranian scholar to give the first known proof of the following well-known identity:

$$
1^{3}+2^{3}+\cdots+n^{3}=(1+2+\cdots+n)^{2} .
$$

Here we give the abstract idea of his method, using today's modern algebraic language. Consider a square array of numbers $M T_{n}=\left(a_{i j}\right)_{1 \leq i, j \leq n}$, as follows

\begin{tabular}{|c||ccccccc|}
\hline$\downarrow i \backslash j \rightarrow$ & 1 & 2 & $\cdots$ & $k$ & $\cdots$ & $n-1$ & $n$ \\
\hline \hline 1 & $a_{11}$ & $a_{12}$ & $\cdots$ & $a_{1 k}$ & $\cdots$ & $a_{1, n-1}$ & $a_{1 n}$ \\
2 & $a_{21}$ & $a_{22}$ & $\cdots$ & $a_{2 k}$ & $\cdots$ & $a_{2, n-1}$ & $a_{2 n}$ \\
$\vdots$ & $\vdots$ & $\vdots$ & $\ddots$ & $\vdots$ & $\vdots$ & $\vdots$ & $\vdots$ \\
$k$ & $a_{k 1}$ & $a_{k 2}$ & $\cdots$ & $a_{k k}$ & $\cdots$ & $a_{k, n-1}$ & $a_{k n}$ \\
$\vdots$ & $\vdots$ & $\vdots$ & $\vdots$ & $\vdots$ & $\ddots$ & $\vdots$ & $\vdots$ \\
$n-1$ & $a_{n-1,1}$ & $a_{n-1,2}$ & $\cdots$ & $a_{n-1, k}$ & $\cdots$ & $a_{n-1, n-1}$ & $a_{n-1, n}$ \\
$n$ & $a_{n 1}$ & $a_{n 2}$ & $\cdots$ & $a_{n k}$ & $\cdots$ & $a_{n, n-1}$ & $a_{n n}$ \\
\hline
\end{tabular}

Our goal is to find the sum of the all entries in two different ways. We denote it by $S_{n}$. First, summing by rows gives us

$$
S_{n}=\sum_{k=1}^{n}\left(\sum_{i=1}^{n} a_{k i}\right)
$$

Then, summing by L-shape pieces (boxed entries in $M T_{n}$ ) results in the following formula for $S_{n}$

$$
S_{n}=\sum_{k=1}^{n}\left(\sum_{i=1}^{k} a_{i k}+\sum_{j=1}^{k} a_{k j}-a_{k k}\right) .
$$

Therefore, from relations (2.1) and (2.2), we get the following general identity:

$$
\sum_{k=1}^{n}\left(\sum_{i=1}^{n} a_{k i}\right)=\sum_{k=1}^{n}\left(\sum_{i=1}^{k} a_{i k}+\sum_{j=1}^{k} a_{k j}-a_{k k}\right) .
$$

From here on, we will call this the $L$-summing equation. In particular, if we choose $a_{i j}=f(i)$ in identity (2.3), we obtain the following one-dimensional specialization of the L-summing equation

$$
\sum_{k=1}^{n}\left(\sum_{i=1}^{k} f(i)+k f(k)\right)=(n+1) \sum_{k=1}^{n} f(k) .
$$

Next, we give several interesting identities which are derived directly from formulas (2.3) and (2.4). Recall that the generalized harmonic numbers $H_{n}^{(s)}$ of order $s$ is defined by $H_{n}^{(s)}=\sum_{k=1}^{n} \frac{1}{k^{s}}$. We also recall that for the complex number $s$, the zeta function is defined as $\zeta(s)=\sum_{k=1}^{\infty} \frac{1}{k^{s}}$. Hence for the natural number $m$, we observe that $\zeta(m)=\lim _{n \rightarrow \infty} H_{n}^{(m)}$.

Example 2.1. By choosing $a_{i, j}=\frac{1}{i^{s}} \times \frac{1}{j^{t}}$ in formula (2.3) for any fixed natural numbers $s$ and $t$, we get the following identity for the generalized harmonic numbers

$$
\sum_{k=1}^{n}\left(\frac{1}{k^{t}} H_{k}^{(s)}+\frac{1}{k^{s}} H_{k}^{(t)}\right)=H_{n}^{(s)} H_{n}^{(t)}+H_{n}^{(s+t)} .
$$


Example 2.2. Letting $f(i)=\frac{1}{k^{s}}(s \in \mathbb{N})$ in formula (2.4), we obtain

$$
\sum_{k=1}^{n} H_{k}^{(s)}=(n+1) H_{n}^{(s)}-H_{n}^{(s-1)}
$$

For more examples, see [2].

Using similar arguments, based on a three dimensional geometric idea and the inclusion - exclusion principle, we can obtain a generalization of L-summing method for three dimensional array $M T_{n}=\left(a_{i j k}\right)_{1 \leq i, j, k \leq n}$.

$$
\begin{aligned}
& \sum_{k=1}^{n} \sum_{j=1}^{n} \sum_{i=1}^{n} a_{i j k} \\
& =\sum_{k=1}^{n}\left(\sum_{i=1}^{k} \sum_{j=1}^{k} a_{k i j}+\sum_{i=1}^{k} \sum_{j=1}^{k} a_{i k j}+\sum_{i=1}^{k} \sum_{j=1}^{k} a_{i j k}\right) \\
& -\sum_{k=1}^{n}\left(\sum_{j=1}^{k} a_{j k k}+\sum_{j=1}^{k} a_{k j k}+\sum_{j=1}^{k} a_{k k j}\right)+\sum_{k=1}^{n} a_{k k k} .
\end{aligned}
$$

In particular, by putting $a_{i j k}=f(i, j)$ in identity (2.5), we get the following two-dimensional specialization

$$
\sum_{k=1}^{n}\left(\sum_{i=1}^{k} \sum_{j=1}^{k} f(i, j)+\sum_{j=1}^{k} k f(k, j)+\sum_{i=1}^{k} k f(i, k)-k f(k, k)\right)=(n+1) \sum_{i=1}^{n} \sum_{j=1}^{n} f(i, j) .
$$

Example 2.3. Choosing $a_{i, j}=\frac{1}{i^{s}} \times \frac{1}{j^{s}} \times \frac{1}{k^{s}}$ in (2.5) for any fixed natural numbers $s$ and considering the definition of the zeta function, after applying the limit operator, we obtain

$$
\sum_{k=1}^{\infty}\left(\frac{\left(H_{k}^{(s)}\right)^{2}}{k^{s}}-\frac{H_{k}^{(s)}}{k^{2 s}}\right)=\frac{\zeta^{3}(s)-\zeta(3 s)}{3} .
$$

Example 2.4. Put $f(i, j)=\left(\begin{array}{l}i-1 \\ j-1\end{array}\right)$ in formula (2.6). Now considering the binomial identity $\sum_{k=0}^{n}\left(\begin{array}{l}n \\ k\end{array}\right)=2^{n}$ and the creative telescoping idea [7], we get

$$
\sum_{k=1}^{n}(k+2) 2^{k-1}=(n+1) 2^{n}-1
$$

\section{The Generalzied Ibn al-Haytham Formula}

A recursive formula for the sums of $k$-th powers of the first $n$ positive integers for $k=4$ for the first time were developed by Ibn al-Haytham, known in Europe as Alhazen [3]. He began by assuming that he knows the formula for $k=3$ (induction hypothesis). Then, using the simple observation that $k+1$ can be written as a sum of $(k+1) 1$ 's or as 2 plus a sum of $(k-1) 1$ 's and so on, he proceeds as follows:

$$
\begin{aligned}
& (k+1)\left(1^{3}+2^{3}+\cdots+(k-1)^{3}+k^{3}\right)= \\
& 1^{3}(1+1+\cdots+1)+2^{3}(2+1+\cdots+1)+\cdots+k^{3}(k+1)= \\
& \left(1^{4}+1^{3}+\cdots+1^{3}\right)+\left(2^{4}+2^{3}+\cdots+2^{3}\right)+\cdots+\left(k^{4}+k^{3}\right),
\end{aligned}
$$

and after using rearrangement technique, he gets

$$
(k+1)\left(\sum_{i=1}^{k} i^{3}\right)=\sum_{i=1}^{k} i^{4}+\sum_{i=1}^{k}\left(\sum_{j=1}^{i} j^{3}\right) .
$$

His interesting elementary idea can be reformulated in general case for sums of $m$-th powers, for any fixed natural number $m$, as follows

$$
(n+1)\left(\sum_{k=1}^{n} k^{m-1}\right)=\sum_{k=1}^{n} k^{m}+\sum_{k=1}^{n}\left(\sum_{i=1}^{k} i^{m-1}\right) .
$$


Using similar argument (see [4]), we can find and prove the following interesting formula for sums of powers of any integer sequence

$$
a_{n+1}\left(\sum_{k=1}^{n} a_{k}^{m-1}\right)=\sum_{k=1}^{n} a_{k}^{m}+\sum_{k=1}^{n}\left(\sum_{i=1}^{k} a_{i}^{m-1}\right)\left(a_{k+1}-a_{k}\right) .
$$

We can even do better, by proving the following more generalized version of Ibn al-Haytham sums of powers formula [4]. From now on, we will call identity (3.1) the generalized Ibn al-Haytham formula.

Theorem 3.1. For any two sequences of real numbers $\left(a_{i}\right)_{i \geq 0}$ and $\left(b_{i}\right)_{i \geq 0}$, we have

$$
a_{n+1}\left(\sum_{k=m}^{n} b_{k}\right)=\sum_{k=m}^{n} b_{k} a_{k}+\sum_{k=m}^{n}\left(\sum_{j=m}^{k} b_{j}\right)\left(a_{k+1}-a_{k}\right), \quad(0 \leq m \leq n) .
$$

For the sake of completeness, we include the proof.

Proof. We can rewrite the left-hand side of (3.1), as follows

$$
\begin{aligned}
a_{n+1}\left(\sum_{k=m}^{n} b_{k}\right) & =\sum_{k=m}^{n} b_{k}\left(a_{n+1}\right)=\sum_{k=m}^{n} b_{k}\left[\left(a_{n+1}-a_{k}\right)+a_{k}\right] \\
& =\sum_{k=m}^{n} b_{k} a_{k}+\sum_{k=m}^{n} b_{k}\left(a_{n+1}-a_{k}\right),
\end{aligned}
$$

therefore, we only need to prove the following identity

$$
\sum_{k=m}^{n} b_{k}\left(a_{n+1}-a_{k}\right)=\sum_{k=m}^{n}\left(\sum_{j=m}^{k} b_{j}\right)\left(a_{k+1}-a_{k}\right) .
$$

By creative telescoping trick [7], we get

$$
a_{n+1}-a_{k}=\sum_{j=k}^{n}\left(a_{j+1}-a_{j}\right)
$$

Hence, we have

$$
\sum_{k=m}^{n} b_{k}\left(a_{n+1}-a_{k}\right)=\sum_{k=m}^{n} b_{k}\left(\sum_{j=k}^{n}\left(a_{j+1}-a_{j}\right)\right) .
$$

Now, interchanging the summation order yields

$$
\sum_{k=m}^{n} b_{k}\left(\sum_{j=k}^{n}\left(a_{j+1}-a_{j}\right)\right)=\sum_{k=m}^{n}\left(\sum_{j=m}^{k} b_{j}\right)\left(a_{k+1}-a_{k}\right) .
$$

Thus, finally, we obtain

$$
a_{n+1}\left(\sum_{k=m}^{n} b_{k}\right)=\sum_{k=m}^{n} b_{k} a_{k}+\sum_{k=m}^{n}\left(\sum_{j=m}^{k} b_{j}\right)\left(a_{k+1}-a_{k}\right),
$$

as required.

As an immediate consequence of the generalized Ibn al-Haytham formula (3.1), we have the following well-known Abel's summation by parts lemma.

Corollary 3.2. For two arbitrary sequences $\left(x_{i}\right)_{i \geq 0}$ and $\left(y_{i}\right)_{i \geq 0}$, we have

$$
\sum_{k=m}^{n-1}\left(x_{k+1}-x_{k}\right) y_{k}=\sum_{k=m}^{n-1} x_{k+1}\left(y_{k}-y_{k+1}\right)+x_{n} y_{n}-x_{m} y_{m}, \quad(m<n) .
$$

In particular, if we choose $b_{k}=\frac{1}{a_{k}^{m}}$ and $m=1$ in formula (3.1), we get the following identity for sums of powers of reciprocals of any integer sequence

$$
a_{n+1}\left(\sum_{k=1}^{n} \frac{1}{a_{k}^{m}}\right)=\sum_{k=1}^{n} \frac{1}{a_{k}^{m-1}}+\sum_{k=1}^{n}\left(\sum_{i=1}^{k} \frac{1}{a_{i}^{m}}\right)\left(a_{k+1}-a_{k}\right) .
$$


Now, if we put $a_{k}=c_{k}^{q-p}, b_{k}=c_{k}^{p}$ and $m=1$ in (3.1) ( $q>p \geq 0$ and both integers), we obtain the following recursive formula which directly relates the sum of $q$-th powers to the sum of $p$-th powers of any integer sequence.

$$
c_{n+1}^{q-p}\left(\sum_{k=1}^{n} c_{k}^{p}\right)=\sum_{k=1}^{n} c_{k}^{q}+\sum_{k=1}^{n}\left(\sum_{i=1}^{k} c_{i}^{p}\right)\left(c_{k+1}^{q-p}-c_{k}^{q-p}\right) .
$$

We also have the following generalized Ibn al-Haytham formula of convolution-type.

Theorem 3.3. For any two sequences of real numbers $\left(a_{i}\right)_{i \geq 0}$ and $\left(b_{i}\right)_{i \geq 0}$, we have

$$
a_{n+1}\left(\sum_{k=m}^{n} b_{k}\right)=\sum_{k=m}^{n} b_{k} a_{n+1-k}+\sum_{k=m}^{n}\left(\sum_{j=m}^{k} b_{n+1-j}\right)\left(a_{k+1}-a_{k}\right), \quad(0 \leq m \leq n) .
$$

Next, we have the following two-dimensional generalization of Ibn al-Haytham sums of powers formula. The proof is similar to the one-dimensional case.

Theorem 3.4. For any two-dimensional array $A=\left(a_{i, j}\right)_{i, j \geq 0}$, we have

$$
\sum_{k=m}^{n} a_{n+1, k}=\sum_{k=m}^{n} a_{k, k}+\sum_{k=m}^{n} \sum_{j=m}^{k}\left(a_{k+1, j}-a_{k, j}\right), \quad(0 \leq m \leq n) .
$$

Corollary 3.5. For any two-variable discrete function $f(x, y)$, we have

$$
\sum_{k=1}^{n} \sum_{i=1}^{k} f(n, i)=\sum_{k=1}^{n} \sum_{i=1}^{k} f(k-1, i)+\sum_{k=1}^{n} \sum_{j=1}^{k} \sum_{i=1}^{j}(f(k, i)-f(k-1, i)) .
$$

Proof. Put $a_{i, j}=\sum_{l=1}^{j} f(i-1, l)$ and $m=1$ in formula (3.2).

Letting $a_{i, j}=a_{i} \cdot b_{j}$ in (3.2), we get the generalized Ibn al-Haytham formula (3.1).

Example 3.6. Putting $f(i, j)=\left(\begin{array}{c}i+j \\ i\end{array}\right)$ in formula (3.3), we obtain the following binomial identity

$$
\sum_{k=1}^{n}\left(\begin{array}{c}
2 k \\
k+1
\end{array}\right)+\sum_{k=0}^{n}\left(\begin{array}{c}
2 k+1 \\
k+1
\end{array}\right)=\left(\begin{array}{c}
2(n+1) \\
n
\end{array}\right)+\left(\begin{array}{c}
n+1 \\
2
\end{array}\right)
$$

In the same spirit of the generalized Ibn al-Haytham formula, we still can come up with another two-dimensional generalization of Ibn al-Haytham formula, as follows.

Theorem 3.7. For any two-dimensional array $A=\left(a_{i, j}\right)_{0 \leq i, j \leq n}$ and arbitrary sequence $\left(b_{k}\right)_{k \geq 0}$, we have

$$
\begin{aligned}
a_{n+1, n+1}\left(\sum_{k=m}^{n} b_{k}\right) & =\sum_{k=1}^{n} b_{k} a_{k, k}+\sum_{i=m}^{n}\left(\sum_{k=m}^{i} b_{k}\right)\left(a_{i+1, n+1}-a_{i, n+1}\right) \\
& +\sum_{j=m}^{n}\left(\sum_{k=m}^{j} b_{k}\right)\left(a_{k, j+1}-a_{k, j}\right), \quad(m \leq n) .
\end{aligned}
$$

Letting $a_{i, j}=a_{i}$ in the identity (3.4), we obtain the standard version of the generalized Ibn al-Haytham formula (3.1).

We finally conclude this section with a three-dimensional generalization of Ibn al-Haytham sums of powers formula.

Theorem 3.8. For any three-dimensional array $A=\left(a_{i, j, k}\right)_{i, j, k \geq 0}$, we have

$$
\sum_{k=m}^{n} \sum_{j=m}^{n} a_{n+1, j, k}=\sum_{k=m}^{n} \sum_{j=m}^{n} a_{k, j, k}+\sum_{i=m}^{n} \sum_{j=m}^{n} \sum_{k=m}^{i}\left(a_{i+1, j, k}-a_{i, j, k}\right), \quad(0 \leq m \leq n) .
$$


The proof is similar to the two-dimensional version and left to the reader as a simple exercise.

Note that by letting $a_{i, j, k}=a_{i, j} \delta_{k j}$ in formula (3.5), where $\delta_{i j}$ is the well - known Kronecker delta symbol and considering the simple trick

$$
\sum_{k=m}^{n} \sum_{j=m}^{n} a_{i, j} \delta_{k j}=\sum_{k=m}^{n} a_{i, k}
$$

we obtain the generalized two - dimensional Ibn al-Haytham formula (3.2). Also, by letting $a_{i, j, k}=a_{i} c_{j} b_{k}$ and plugging into formula (3.5), we get the generalized Ibn al-Haytham formula (3.1).

Corollary 3.9. For any two-dimensional array $A=\left(a_{i, j}\right)_{i, j \geq 0}$ and arbitrary sequence $\left(b_{k}\right)_{k \geq 0}$, we have

$$
\left(\sum_{k=m}^{n} b_{k}\right) \sum_{j=m}^{n} a_{n+1, j}=\sum_{k=m}^{n} b_{k}\left(\sum_{j=m}^{n} a_{k, j}\right)+\sum_{i=m}^{n} \sum_{j=m}^{n}\left(\sum_{k=m}^{i} b_{k}\right)\left(a_{i+1, j}-a_{i, j}\right), \quad(0 \leq m \leq n) .
$$

In particular, for any two-variable discrete function $(f(i, j))_{i, j \geq 0}$, we have

$$
(n+1) \sum_{j=0}^{n} \sum_{i=0}^{n} f(i, j)=\sum_{k=0}^{n} \sum_{j=0}^{n}\left(\sum_{l=0}^{k} f(l, j)\right)+\sum_{k=0}^{n} \sum_{j=0}^{n} k f(k, j)
$$

Example 3.10. Putting $a_{i, j}=\left(\begin{array}{c}i-1 \\ j-1\end{array}\right)$ and $b_{k}=\frac{1}{k}$ in equation (3.6), for $m=1$, we obtain

$$
\sum_{k=1}^{n} 2^{k-1} H_{k}+\sum_{k=1}^{n} 2^{k-1} \frac{1}{k}=\left(2^{n}-1\right) H_{n}
$$

which may be interesting in the light of the following well-known identity

$$
\sum_{k=1}^{n} 2^{k-1}+\sum_{k=1}^{n} 2^{k-1}=2^{n+1}-1
$$

\section{Karaji's Method Versus Ibn al-Haytham Formula}

At the end of this paper, as we mentioned in the introduction, we intend to show the interesting interplay between two seemingly different summation techniques that we already mentioned in the previous sections.

We first note that by choosing $a_{i j}=b_{i}\left(a_{j+1}-a_{j}\right)$ in L-summing equation (2.3) and considering the creative telescoping technique, we get

$$
\begin{aligned}
\sum_{k=1}^{n}\left(\sum_{i=1}^{n} b_{k}\left(a_{i+1}-a_{i}\right)\right) & =\sum_{k=1}^{n}\left(\sum_{i=1}^{k} b_{i}\left(a_{k+1}-a_{k}\right)+\sum_{j=1}^{k} b_{k}\left(a_{j+1}-a_{j}\right)-b_{k}\left(a_{k+1}-a_{k}\right)\right) \\
\sum_{k=1}^{n} b_{k}\left(\sum_{i=1}^{n}\left(a_{i+1}-a_{i}\right)\right) & =\sum_{k=1}^{n}\left(\left(\sum_{i=1}^{k} b_{i}\right)\left(a_{k+1}-a_{k}\right)+b_{k} \sum_{j=1}^{k}\left(a_{j+1}-a_{j}\right)-b_{k}\left(a_{k+1}-a_{k}\right)\right) \\
\sum_{k=1}^{n} b_{k}\left(a_{n+1}-a_{1}\right) & =\sum_{k=1}^{n}\left(\sum_{i=1}^{k} b_{i}\right)\left(a_{k+1}-a_{k}\right)+\sum_{k=1}^{n} b_{k}\left(a_{k+1}-a_{1}\right)-\sum_{k=1}^{n} b_{k}\left(a_{k+1}-a_{k}\right) \\
a_{n+1}\left(\sum_{k=1}^{n} b_{k}\right) & =\sum_{k=1}^{n} b_{k} a_{k}+\sum_{k=1}^{n}\left(\sum_{i=1}^{k} b_{i}\right)\left(a_{k+1}-a_{k}\right),
\end{aligned}
$$

which is the generalized Ibn al-Haytham formula (3.1) for $m=1$.

Now, letting $a_{i j}=m_{j+1, i}-m_{j, i}$ in L-summing equation (2.3) and doing similar calculations, we obtain the generalized two-dimensional Ibn al-Haytham formula (3.2) for the array $M=\left(m_{i, j}\right)_{i, j \geq 1}$.

In the reverse direction, we first note that by putting $a_{i, j}=\sum_{l=1}^{i-1} f(j, l),(m=1)$ in identity (3.2) and considering the fact 
that $a_{k+1, j}-a_{k, j}=f(j, k)$ we have

$$
\begin{aligned}
\sum_{k=1}^{n} \sum_{l=1}^{n} f(k, l) & =\sum_{k=1}^{n} \sum_{l=1}^{k-1} f(k, l)+\sum_{k=1}^{n} \sum_{j=1}^{k} f(j, k), \\
\sum_{k=1}^{n} \sum_{l=1}^{n} f(k, l) & =\sum_{k=1}^{n}\left(\sum_{l=1}^{k} f(k, l)-f(k, k)\right)+\sum_{k=1}^{n} \sum_{j=1}^{k} f(j, k), \\
\sum_{k=1}^{n} \sum_{l=1}^{n} f(k, l) & =\sum_{k=1}^{n}\left(\sum_{j=1}^{k} f(j, k)+\sum_{l=1}^{k} f(k, l)-f(k, k)\right),
\end{aligned}
$$

which is exactly the two-dimensional L-summing equation (2.3). It is also worth to note that by choosing $a_{k}=k$, $b_{k}=f(k)$ and $m=1$, we conclude the one-dimensional specialization of Karaji's L-summing formula (2.4).

It seems that above arguments show that despite of the fact that these two folkloric summation techniques have different natures, Karaji's L-summing method is based on the geometric idea and the generalized Ibn al-Haytham formula has the recursive nature, but they can transform to each other by some parametric transformations.

Remark 4.1. Using the generalized Ibn Haytham and its higher dimensional extensions and the L - summing techniques, one can systematically find and prove some general classes of integer sequences identities. This is the next research project of the author [5] in the area of algebraic identities.

\section{CONFLICTS OF INTEREST}

The author declares that there are no conflicts of interest regarding the publication of this article.

\section{REFERENCES}

[1] Greene, D. H., Knuth, D. E., Mathematics for the Analysis of Algorithms (3rd Edition), Birkhauser, Boston, 1990.

[2] Hassani, M., Identities by L-summing method, Int. J. Math. Comput. Sci., 1(2006), 165-172.

[3] Katz, V. J., Ideas of calculus in Islam and India, Math. Mag., 68(1995), 163-174.

[4] Teimoori, H., A Generalization of Ibn Al-Haytham recursive formula for sums of powers, Turk. J. Math. Comput. Sci., 1(2018), 165-172.

[5] Teimoori, H. A Systematic way to prove some classes of integer sequences identities, (in preparation).

[6] Woepcke, F., Extrait du Fakhr, Traite D'algebre par Abou Bekr Mohammed Ben Alhacan Alkarkhi, Reprint of the 1853 original, Hildesheim, 1982.

[7] Zeilberger, D., The method of creative telescoping, J. Symbolic Comput., 11(1991), 195-204. 